\title{
Hybrid near-field scanning optical microscopy tips for live cell measurements
}

\author{
Luka K. Kapkiai, David Moore-Nichols, Jonathan Carnell, Jeffrey R. Krogmeier, ${ }^{\text {a) }}$ \\ and Robert C. Dunn ${ }^{b}$ \\ Department of Chemistry, University of Kansas, Malott Hall, Lawrence, Kansas 66049
}

(Received 12 January 2004; accepted 8 March 2004; published online 29 April 2004)

\begin{abstract}
We report a near-field scanning optical microscopy (NSOM) probe that enables high-resolution imaging of living cells under physiological buffered conditions. The hybrid design combines a conventional fiber optic near-field probe with a standard atomic force microscopy cantilever. Imaging of fluorescent latex spheres suspended in an acetate matrix demonstrates the subdiffraction limited fluorescence and topography capabilities of the tips. The reduced spring constant of the hybrid tip is also shown to be amenable to measurements on living cells. NSOM fluorescence and topography measurements on living human arterial smooth muscle cells under buffered conditions are demonstrated. (C) 2004 American Institute of Physics. [DOI: 10.1063/1.1737464]
\end{abstract}

Near-field scanning optical microscopy (NSOM) is a scanning probe technique that utilizes specially fabricated probes to deliver light down to the nanometric dimension. ${ }^{1,2}$ NSOM provides opportunities to simultaneously measure both optical and topographic features of samples with subdiffraction limited spatial resolution. These capabilities have been utilized to probe thin films, solid-state devices, and due to the unique electromagnetic fields emerging from the nearfield aperture, single molecule orientations. ${ }^{2}$ The potential impact of NSOM is arguably the greatest in the biological sciences, where there is a well-developed history of using fluorescence probes to tag specific proteins or structures. This field seems particularly well suited to take advantage of the single molecule fluorescence sensitivity, high spatial resolution, and simultaneous force information that NSOM offers. However, current applications of NSOM to biological samples are largely limited to isolated protein samples, model membranes, or chemically fixed biological cells. ${ }^{2-5}$ The extension to viable, unfixed biological tissues has previously proven problematic.

The difficulty in conducting NSOM measurements on viable biological tissues arises from the forces generated in maintaining the NSOM tip close to the specimen. Highresolution NSOM measurements require that the tip be held within nanometers of the sample while scanning. This necessitates the implementation of a feedback system for sensing the sample surface and maintaining the tip-sample gap. ${ }^{6-8}$ Traditionally, a force feedback approach is implemented in which the tip is either dithered laterally or vertically to the sample surface, depending on probe geometry. The dampening in the amplitude of the oscillating NSOM tip as the tip interacts with the sample surface is monitored and used to hold the tip-sample gap constant during scanning. While straightforward and highly successful for most applications, the large forces generated using conventional fiber optic

\footnotetext{
${ }^{a)}$ Current address: National Institute of Standards and Technology, 100 Bureau Drive Mail Stop 8443, Gaithersburg, MD 20899-8441.

b) Author to whom correspondence should be addressed; electronic mail: rdunn@ku.edu
}

NSOM probes often damage fragile biological samples such as living cells.

A number of approaches have been reported in attempts to circumvent the large forces generated under normal NSOM feedback operation and thus open applications in the biological sciences. These have largely involved either changing the feedback mechanism utilized to hold the tip close to the sample or modifying the probes themselves to lower the spring constant and thus the forces generated in force feedback. ${ }^{2,5,9}$ For the latter, an obvious route of exploration is to incorporate a near-field light source onto conventional atomic force microscopy (AFM) cantilevers. ${ }^{9-11}$ AFM is widely used in biological applications on unfixed tissues under physiological conditions.

Commercially available silicon nitride AFM cantilevers come in a variety of geometries that offer a range of cantilever spring constants and tip parameters. Using these tips, AFM techniques have been widely utilized for studies of sample surfaces, including living cells, while operating with the AFM tip either directly in contact with the sample surface or through a tapping, or intermittent, contact mode of force mapping. ${ }^{12}$ Though AFM has been successful in imaging living cells, a robust chemical contrast mechanism is not easily incorporated and often limits the technique to topographical information.

Previously, we have shown that focused ion beam (FIB) micromachining of commercial AFM tips could be used to fabricate a near-field light source on an AFM cantilever. ${ }^{9}$ The FIB was used to cut a small hole into the end of an AFM tip, into which a high index glass sphere $(\eta=1.9)$ was glued. The glass sphere was then milled into a pyramid shape with the FIB and the sides were coated with aluminum to confine the light inside the taper region. A small aperture was opened at the very end of the pyramid, which served as the near-field light source. Advantages of these tips include a reduced spring constant and ability to incorporate high index materials, such as GaP, to improve the performance. The tips suffer, however, from an intensive fabrication process and variable aperture properties. Sculpting the spheres into pyramid structures is time consuming and because of the pyramid geom- 


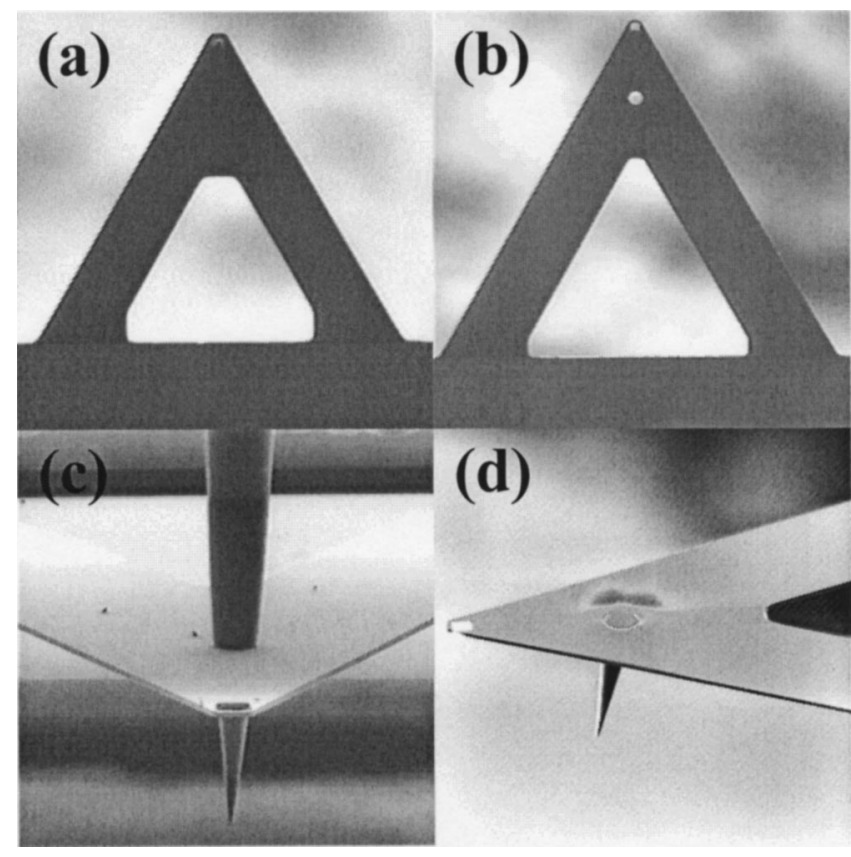

FIG. 1. (A) High-resolution electron microscopy image of a conventional silicon nitride AFM cantilever with a spring constant of $0.12 \mathrm{~N} / \mathrm{m}$. (B) The FIB is used to cut a $7 \mu \mathrm{m}$ hole in the end of the cantilever. (C) A fiber optic NSOM probe is carefully lowered through the hole and glued into place. (D) The FIB is used to cut the excess fiber from the backside of the AFM cantilever and polish the fiber smooth with the cantilever surface.

etry, the apertures are not circularly symmetric. Both of these are technical problems which can be addressed with more sophisticated instrumentation, but here we demonstrate an alternative solution based on incorporating conventional fiber optic NSOM probes into AFM cantilvers.

Figures 1(A)-(D) demonstrate the process developed to fabricate hybrid NSOM/AFM probes incorporating conventional fiber optic NSOM tips. Figure 1(A) shows a silicon nitride AFM cantilever (Veeco Instruments Inc., $0.12 \mathrm{~N} / \mathrm{m}$ spring constant) in which a $7 \mu \mathrm{m}$ hole is cut with the FIB [Fig. 1(B)]. Fiber optic NSOM probes are fabricated using standard techniques. ${ }^{1}$ Briefly, a single-mode optical fiber
$(3 \mathrm{M})$ is heated and pulled to a fine point using a commercial micropipette puller (Sutter Instruments). The pulled fibers are then evaporatively coated with approximately 50-100 $\mathrm{nm}$ of aluminum around the taper region in a custom designed evaporation chamber. The aluminum coating confines the light in the taper of the probe such that it only exits at the aperture of the tip apex.

The fiber optic NSOM tip is carefully lowered using a hydraulic $x y z$ positioner (Narishige International Inc.) through the hole cut in the end of the AFM cantilever. The fiber optic NSOM tip is affixed in place with glue [Fig. 1(C)] and the assembly is then placed back into the FIB to machine off the excess fiber from the backside of the cantilever as shown in Fig. 1(D). It is essential to cut the fiber flush with the reflecting surface of the AFM cantilever to achieve both a good reflection, used in generating the feedback signal, and efficient coupling of light into the NSOM tip.

The hybrid fiber optic NSOM/AFM tips are mounted in a conventional Dimension 3000 AFM head (Digital Instruments), which has been modified so that an external light source can be delivered to the tip. For the experiments described here, the $514 \mathrm{~nm}$ line from an argon ion source (Liconix, 5000 series) is focused onto the backside of the AFM cantilever and serves as both the excitation source through the NSOM tip and to generate the force feedback signal, as previously reported. ${ }^{9}$ The sample is mounted on $x-y$ closed-loop piezo stage (Mad City Labs, Inc.) and used to raster scan the sample beneath the probe. Fluorescence is collected beneath the sample with a high numerical aperture objective lens (Zeiss, Fluar 100X, 1.3 NA), appropriately filtered, and imaged onto an avalanche photodiode (EG\&G, SPCM-200). All aspects of the imaging system are controlled with a Nanoscope IIIa controller (Digital Instruments).

Figure 2 shows NSOM (A) fluorescence and (B) topography images of a standard sample consisting of $50 \mathrm{~nm}$ fluorescent latex spheres (Duke Scientific Corporation) embedded in an acetate matrix, taken with a hybrid fiber optic NSOM/AFM tip. Unlike conventional NSOM measurements employing fiber optic probes, the reduced spring constant of
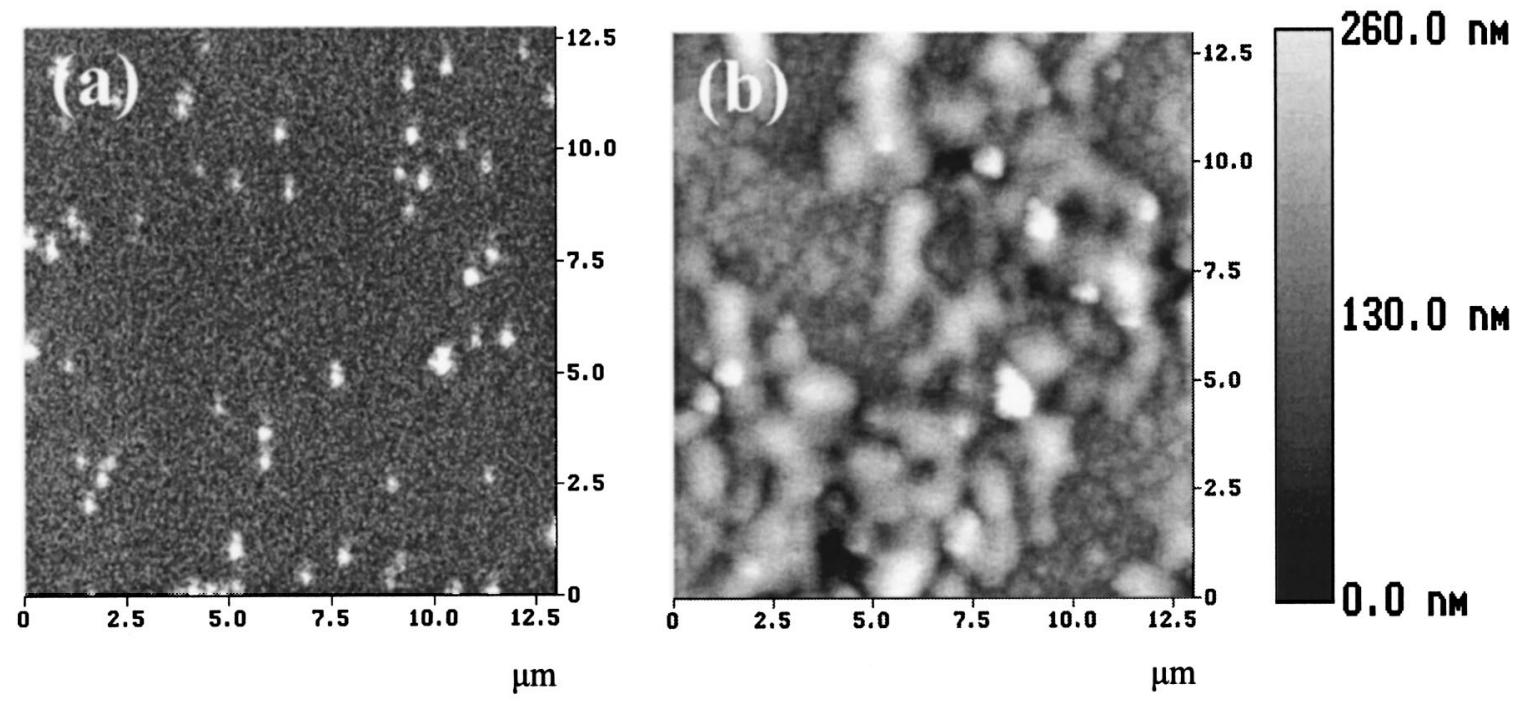

FIG. 2. Simultaneous (A) NSOM fluorescence and (B) topography images of $50 \mathrm{~nm}$ fluorescent latex spheres embedded in an acetate matrix taken with the fiber optic NSOM/AFM tip hybrid. The full width at half maximum of the smallest features in the fluorescence image corresnonds to 130 nm, representing the convolution between the aperture size and the $50 \mathrm{~nm}$ spheres. 


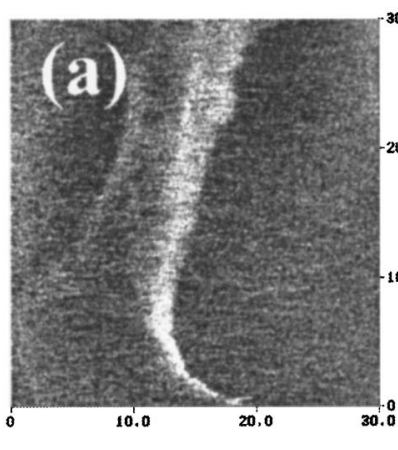

$\mu \mathrm{m}$

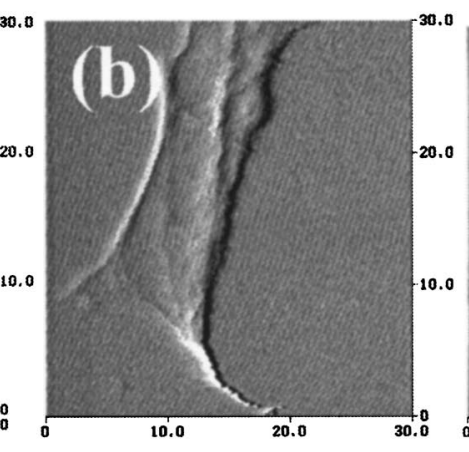

$\mu \mathrm{m}$

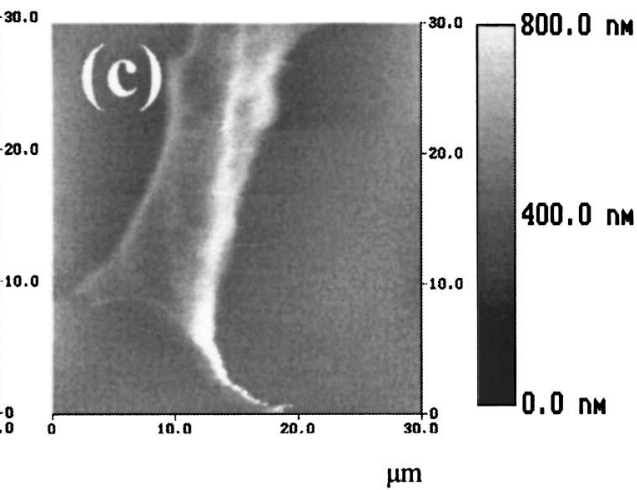

$\mu \mathrm{m}$

FIG. 3. (A) Near-field fluorescence (B) feedback error signal and (C) topography images of a living human arterial smooth muscle cell cultured on a glass coverslip taken under buffered conditions. Adrenergic receptors have been fluorescently labeled with prazosin BODIPY-FL. The images were collected in contact mode and repeated scans of the same cell revealed no evolution in the cell morphology, illustrating the noninvassive nature of the hybrid tips.

the hybrid tips allows for contact mode measurements to be conducted without damaging the tip aperture or sample. This mode is more stable than tapping-mode feedback and is beneficial for NSOM measurements since the tip remains near the sample surface where resolution is maximized. From the features seen in the NSOM fluorescence image, a resolution of $130 \mathrm{~nm}$ is determined, demonstrating the subdiffraction capabilities of the hybrid tip.

As stated earlier, the real motivation for fabricating NSOM sources on AFM cantilevers is to open applications in the biological sciences on unfixed, viable tissues, which has remained problematic for NSOM. To demonstrate the live cell imaging capabilities of these hybrid NSOM/AFM tips, Figs. 3(A)-3(C) show the simultaneously measured NSOM fluorescence, feedback error signal, and topography images, respectively, of a living human arterial smooth muscle (HASM) cell under buffered conditions. The HASM cells were cultured on glass coverslips using standard techniques and adrenergic receptors in the cell membrane were fluorescently labeled with prazosin BODIPY-FL (Molecular probes). The cells were not exposed to any chemical fixatives and were imaged at room temperature under Hanks HEPES modified buffer using the hybrid NSOM/AFM probes in contact mode. No evolution in the cellular topography was observed following multiple scans, indicating that tip-sample forces did not damage the fragile cell membrane. In addition to tracking the HASM cell topography, subdiffraction limited optical features on the order of $130 \mathrm{~nm}$ are resolved. The simultaneous topography and optical imaging of live HASM cells demonstrates the utility of these probes for biological applications and represents a high-resolution tool in studying cell membrane activities.

The successful demonstration of live cell fluorescence imaging with the hybrid fiber optic NSOM/AFM probes opens many applications in the biological sciences that can benefit from the high-resolution fluorescence and topography capabilities. These tips incorporate the well-known properties of fiber optic NSOM probes and are much less rigorous to fabricate than the previous hybrid design. ${ }^{9}$ While still requiring access to FIB technology, the micromachining requirements are greatly relaxed since fabrication of the aperture itself is not required. Moreover, since these tips are designed around conventional fiber optic NSOM probes, they take advantage of the past work done in fabricating and understanding the optical properties of these tips. Now that NSOM can be implemented in viable tissues, the low detection limits and high spatial resolution should provide a useful tool for studying nanometric structures in the cellular membrane, such as lipid rafts, that to date have proven elusive to probe directly.

J.R.K. gratefully acknowledges support from the NIH Dynamic Aspects of Chemical Biology predoctoral training grant. The authors gratefully acknowledge support from NIH (GM55290) and the Madison and Lila Self Foundation.

${ }^{1}$ E. Betzig, J. K. Trautman, T. D. Harris, J. S. Weiner, and R. L. Kostelak, Science 251, 1468 (1991).

${ }^{2}$ R. C. Dunn, Chem. Rev. (Washington, D.C.) 99, 2891 (1999).

${ }^{3}$ H. Muramatsu, N. Chiba, K. Homma, K. Nakajima, T. Ataka, S. Ohta, A. Kusumi, and M. Fujihira, Appl. Phys. Lett. 66, 3245 (1995).

${ }^{4}$ J. Hwang, L. A. Gheber, L. Margolis, and M. Edidin, Biophys. J. 74, 2184 (1998).

${ }^{5}$ M. Koopman, B. I. de Bakker, M. F. Garcia-Parajo, and N. F. van Hulst, Appl. Phys. Lett. 83, 5083 (2003).

${ }^{6}$ K. Karrai and R. D. Grober, Appl. Phys. Lett. 66, 1842 (1995).

${ }^{7}$ R. Brunner, O. Hering, O. Marti, and O. Hollricher, Appl. Phys. Lett. 71, 3628 (1997)

${ }^{8}$ C. E. Talley, G. A. Cooksey, and R. C. Dunn, Appl. Phys. Lett. 69, 3809 (1996).

${ }^{9}$ J. R. Krogmeier and R. C. Dunn, Appl. Phys. Lett. 79, 4494 (2001).

${ }^{10}$ R. Eckert, J. M. Freyland, H. Gersen, H. Heinzelmann, G. Schurmann, W. Noell, U. Staufer, and N. F. de Rooij, Appl. Phys. Lett. 77, 3695 (2000).

${ }^{11}$ P. N. Minh, T. Ono, and M. Esashi, Rev. Sci. Instrum. 71, 3111 (2000).

${ }^{12}$ J. A. Dvorak, Methods 29, 86 (2003). 